\title{
Small-sized rectangular liquid-filled acoustical tank excitation: a modal approach including leakage through the walls
}

\author{
Antonin Novak ${ }^{1}$, Michel Bruneau, Pierrick Lotton \\ Laboratoire d'Acoustique de I'Université du Mans, LAUM - UMR 6613 CNRS, Le Mans Université, Avenue Olivier \\ Messiaen, 72085 LE MANS CEDEX 9, France \\ *antonin.novak@univ-lemans.fr \\ *https://ant-novak. com
}

\begin{abstract}
Small-sized water-filled tanks with thin (compared to the acoustic wavelength) walls that are surrounded on all sides by air, have been used over the past decades for underwater studies. So far, the analytical modelling of the acoustic field inside such an enclosure considers either the eigenvalue problem with pressure release conditions at the walls or imposes empirical impedance boundary conditions, or even proceeds in analogy with the field within a waveguide. At low frequencies, approximations involving an incompressible fluid or the Laplace equation have been used. Those models have limitations that are always caused by simplification of the boundary conditions. This paper deals with both an analytical formulation expressing the acoustic leakage through the walls (lossy and reacting walls) and modal solutions for the acoustic pressure and acoustic velocity fields when a source emits energy and when it is shut off, providing the transient acoustic response of small tanks quantitatively. Several analytical results are compared with experimental observations.
\end{abstract}

The archived file is not the final published version of the article A. Novak, M. Bruneau, \& P. Lotton (2018), "Small-Sized Rectangular Liquid-Filled Acoustical Tank Excitation: A Modal Approach Including Leakage Through the Walls", Acta Acustica united with Acustica. Vol. 104(4), pp. 586-596.

The definitive publisher-authenticated version is available online at $\mathrm{http} / / \mathrm{dx} . \mathrm{doi} . \mathrm{org} / 10.3813 / \mathrm{AAA} .919199$, Readers must contact the publisher for reprint or permission to use the material in any form. 


\section{Introduction}

Over the past decades, many underwater studies in small-sized tanks of water, with thin walls (compared to the acoustic wavelength) that are surrounded on all sides by air, have been performed [1,2]. An analytical modeling procedure that is suitable to characterize the sound emitted and/or received at any location inside such a small tank is needed. So far, on one hand, the analytical modeling of the acoustic field (pressure and particle velocity) inside the tank assumes that the tank behaves approximately as a waveguide or assumes that the damping due to the reflections from the six surfaces of the rectangular "brick" of water is negligible. In both cases, only eigenvalue problems below the first resonant frequency normal mode are considered [1, 3, 4, among others]. Such analyses assume an incompressible fluid or the Laplace equation in the lower frequency range [5]. On the other hand, a numerical simulation based on the finite difference method gives results that agree only qualitatively with experimental ones (due to the simplistic treatment of the boundary conditions), below and above the lowest resonant frequency [6]. Also, an analytical and numerical approach, assuming normal plane wave modelling in the elastic walls and neglecting the radiation outside the tank, provides a method for predicting the acoustic field in tanks [7]. Although the latter two models are improvements over the previous ones, they have limitations that are due to the assumptions involved in the boundary condition specifications. Therefore, a better analytical insight into the effects of the walls on the acoustic fields within the tank, which account for leakage through the walls modelled with a more realistic field inside the elastic walls, may be helpful to obtain the acoustic response of small tanks quantitatively. Note that sources considered in such modelling are assumed to be point sources so they do not alter the modal behaviour of the tank. It is clear that an extended source (e.g., a swim bladder in very small tank) may have to be considered explicitly in the formalism as a vibrating boundary within the domain. Consideration of such extended sources are beyond the scope of the paper.

In other words, until now, regarding the theoretical interpretations of the results for experiments carried out in small tanks, more often the analytical approaches available in the literature rely on standard eigenvalue solutions that assume the acoustic pressure decays to zero at the walls, the bottom, and the free-surface of the "brick" of liquid. The orthonormal eigenfunctions $\psi_{m}^{(0)}(x, y, z)$, associated to the pressure field, that are solutions for the set of equations including a Helmholtz equation and Dirichlet boundary conditions, take the well-known form $(m$ representing the triplet of indices $\left.\left(m_{x}, m_{y}, m_{z}=1,2,3, \ldots\right)\right)$

$$
\begin{aligned}
\psi_{m}^{(0)}(x, y, z)= & \sqrt{\frac{2^{3}}{L_{x} L_{y} L_{z}}} \sin \left(k_{m_{x}}^{(0)} x\right) \\
& \cdot \sin \left(k_{m_{y}}^{(0)} y\right) \sin \left(k_{m_{z}}^{(0)} z\right),
\end{aligned}
$$

where $L_{x}, L_{y}$, and $L_{z}$ are the dimensions the tank?s interior, and where the eigenvalues $k_{m}^{(0)}$ are given by

$$
\begin{array}{r}
\left(k_{m}^{(0)}\right)^{2}=\left(k_{m_{x}}^{(0)}\right)^{2}+\left(k_{m_{y}}^{(0)}\right)^{2}+\left(k_{m_{z}}^{(0)}\right)^{2}, \\
k_{m_{x}}^{(0)}=\frac{m_{x} \pi}{L_{x}}, \quad k_{m_{y}}^{(0)}=\frac{m_{y} \pi}{L_{y}}, \quad k_{m_{z}}^{(0)}=\frac{m_{z} \pi}{L_{z}},
\end{array}
$$

showing that the total acoustic pressure vanishes at the walls. The components of the particle velocity obtained from the Euler equation $\rho \partial_{t} \vec{v}=-\vec{\nabla} p$, are proportional to

$$
\begin{aligned}
\left(v_{x}\right)_{m}(x, y, z) & \propto k_{m_{x}}^{(0)} \cos \left(k_{m_{x}}^{(0)} x\right) \\
& \cdot \sin \left(k_{m_{y}}^{(0)} y\right) \sin \left(k_{m_{z}}^{(0)} z\right),
\end{aligned}
$$

(and similarly, for the components $v_{y}$ and $v_{z}$ ). This shows that the particle velocity is normal to each wall at any location on the wall considered (the components parallel to the wall vanish at the wall under consideration). 
Let us note that, in accordance with this approximation, the six surfaces are assumed to be perfect sound reflectors and the damping due to the numerous reflections per unit time is neglected. Actually, this "zero order" approximation leads to infinite values for the modal quality factors; this is unfortunately not physically realistic.

As mentioned in the literature cited earlier and as shown in the following section, in actual situations, the boundary conditions at the upper surface of the liquid (water) and at the walls of the tank (including the tank floor) correspond to non-zero (but small) acoustic pressure amplitudes associated with a particle movement that exist even close to the wall. Therefore, when considering such slightly absorbing (due to the acoustic energy radiated outside the tank) and reacting vibrating walls, a quite different distribution of pressure variation and particle velocity is modelled analytically by a quite simple modal approach which leads to accurate quantitative results when an acoustic source provides energy in the medium or when it is removed (or shut off). The present paper deals with such analytical modelling. Considering in this modelling that precise theoretical approach of the complex coupling between the liquid and a solid boundary of finite sizes would prevent us from obtaining a tractable analytical model, a lossy and reacting impedance-like behaviour of the boundaries, obtained from a multi-layered approach, is suggested herein. This impedance-like behaviour includes both the effects of the radiative energy leakage previously mentioned(the main effect) and also a relevant empirically determined very small damping parameter inside the wall itself.

Since there exist a vast amount of literature concerned with boundary problems involving the Helmholtz equation and locally reacting boundary conditions $[8,9,10,11,12,13,14,15,16,17$, among others], no claims are here made as to fundamental novelty in the sense that the present study relies on standard formulations based on the classical equations of acoustics, but this paper does provide appropriate analytical modelling and reliable results. Therefore, such a specific paper dedicated to researchers that perform acoustical studies in small liquid-filled tanks should be of some interest and value, both below and above the lowest resonant frequency. Note that when considering applications that take advantages of the energy distributed in the first resonances (up to $10 \mathrm{kHz}$ herein) by sources (small fishes or shrimp for example), it should be important to have an analytic modelling to explain sound pressure distribution in a tank quantitatively in a frequency range which contains these resonances.

This paper is divided into three parts: An approximate multi-layered analytical approach to interpret both the impedance-like behaviour of the walls and the free upper surface of the liquid are presented in Section 2. Then the analytical solutions inside the tank with lossy and reacting walls are presented; first, the set of standing waves (Section 3) and second, the modal solutions when a point source is providing energy (Section 4). Finally, the last section (Section 5) presents a comparison between the proposed analytical results and experimental measurements.

\section{Approximate analytical approach for the reflection coefficient of the walls}

To find an analytical expression of the reflection coefficient for any wall, it is appropriate to first write the general form of the modal wave functions within the liquid that satisfy realistic boundary conditions. The modal solutions (1) that satisfies the Dirichlet conditions is a particularly simple case. This general form of the modal wave functions that govern the spatial variation of the acoustic field inside the tank can be written as

$$
\begin{aligned}
\psi_{m}(x, y, z)= & A_{m}\left(e^{-i k_{m_{x}} x}+B_{m_{x}} e^{i k_{m_{x}} x}\right) \\
& \cdot\left(e^{-i k_{m_{y}} y}+B_{m_{y}} e^{i k_{m_{y}} y}\right) \\
& \cdot\left(e^{-i k_{m_{z}} z}+B_{m_{z}} e^{i k_{m_{z}} z}\right),
\end{aligned}
$$

where $m$ stands for the triplet of indices $\left(m_{x}, m_{y}, m_{z}=\right.$ $0,1,2, \ldots)$ and where the coefficients (integration constants) $B_{m_{x}}, B_{m_{y}}$, and $B_{m_{z}}$ are here unknown although they are expressed in equation Eq. (17).

The model presented here to express the reflection coefficient at a wall relies basically on the model of the movement of a thick plate $[9$, chapter 8$]$ that has been adapted to a thin plate loaded by water at its inner surface 
and by air at its outer surface. That approach accounts for the modal nature (nearly Dirichlet) of the incident wave (forced movement) and leads to the boundary conditions of a supported plate (without movement at its edges, as demonstrated below).

To be as simple as possible, it assumes in addition that the shear movement (more generally the in-plane $\left(u^{\prime}, u^{\prime \prime}\right)$ movement) can be neglected because this movement cannot be exited by the movement of the liquid at the interface. The shear stress is assumed to vanish at the interface liquid-solid) and anyway it cannot be responsible for energy transfer to the air which is assumed to be inviscid (i.e., no "shear leakage" through the wall). Neglecting both the shear movement and the shear stress (as mentioned above), and in addition the bending moment (i.e. assuming a forced flexural profile, see details below), the only normal stress is accounted for in the behaviour of the wall (the stress tensor is diagonal and the strain tensor reduces to the displacement along the u-axis). Therefore the parameters which characterize the wall are (among the density) the Young modulus and the Poisson's ratio which are accounted for in the velocity $c_{w}$ of the disturbance. Then, the basic equation which relates the velocity field to the normal stress reduces to the classical Newton's law (or equivalently here the Euler's equation) which relates the particle velocity to the derivative with respect to the variable $u$ of the normal stress (namely here the pressure variation), the only one considered here in an approach as simple as possible. Note that the Hooke's law could apply actually, but it involves the spatial derivatives of the displacement field in the lateral directions $u^{\prime}$ and $u^{\prime \prime}$ as well $\left(k_{m_{u}}^{(w)} \neq k_{m}^{(w)}\right)$ which is not considered here in the frame of the approximation mentioned above (see for example [9] equation 8.131). Finally, the reflection coefficient obtained enlightens the absorption and reaction behaviour of the wall, the absorption being due to the energy transfer from the water to the air through the wall ("compressional leakage" which is the main factor of dissipation of energy in the tank).

In order to characterize this behaviour (modal reflection coefficient or equivalently modal impedance-like), we consider a wall with an outward (i.e. directed towards the wall) local $u$ - axis, the coordinate of the inner and the outer planar surface of the wall being respectively $u=0$ and $u=h$ ( $h$ denoting the thickness of the wall). The wall considered is labelled $u_{j}(j=1,2$ denoting both walls perpendicular to the $u$ - axis) and the other coordinates are denoted $u^{\prime} \in\left(0, L_{u^{\prime}}\right)$ and $u^{\prime \prime} \in\left(0, L_{u^{\prime \prime}}\right)$. The subscripts $w$, and $a$ denote respectively the wall and the air outside the tank (the subscript $l$ denoting the liquid is removed in order to simplify the writing). The pressure wave function inside the tank, in the wall, and in the air outside the tank, can be written as follows, with $\omega_{m}=k_{m} / c=k_{m}^{(w)} / c_{w}=k_{m}^{(a)} / c_{a}$,

$$
p_{m}(u, t)=A\left(e^{-i k_{m_{u}} u}+R_{u_{j} m} e^{i k_{m_{u}} u}\right) \phi_{\mu_{u}}\left(u^{\prime}, u^{\prime \prime}\right) e^{i \omega_{m} t}
$$

where $R_{u_{j} m}$ is the reflection coefficient and where the function $\phi_{\mu_{u}}\left(u^{\prime}, u^{\prime \prime}\right)$ represents the modal solution for the acoustic field along the coordinates $u^{\prime}$ and $u^{\prime \prime}$ (transversal modes, $\mu_{u}$ standing for the couple $m_{u}^{\prime}$ and $m_{u}^{\prime \prime}$ ),

$$
\begin{aligned}
p_{m}^{(w)}(u, t)= & {\left[C_{w} \cos \left(k_{m_{u}}^{(w)} u\right)+D_{w} \sin \left(k_{m_{u}}^{(w)} u\right)\right] } \\
& \cdot \phi_{\mu_{u}}^{(w)}\left(u^{\prime}, u^{\prime \prime}\right) e^{i \omega_{m} t}, \\
p_{m}^{(a)}(u & =h, t)=A_{a} \phi_{\mu_{u}}^{(a)}\left(u^{\prime}, u^{\prime \prime}\right) e^{i \omega_{m} t},
\end{aligned}
$$

where $\phi_{\mu_{u}}^{(w)}\left(u^{\prime}, u^{\prime \prime}\right), \phi_{\mu_{u}}^{(a)}\left(u^{\prime}, u^{\prime \prime}\right), k_{m_{u}}^{(w)}$, and $k_{m_{u}}^{(a)}$ are functions to be determined, $(\rho$ and $c),\left(\rho_{w}\right.$ and $\left.c_{w}\right)$, and $\left(\rho_{a}\right.$ and $c_{a}$ ) being the density and the speed of sound in the liquid, the wall, and the air respectively.

The continuity of both pressure variation and normal particle velocity (obtained from Euler equation $\rho \partial_{t} \vec{v}=$ $-\vec{\nabla} p)$ at the interfaces leads readily to the modal specific impedance-like $\zeta_{u_{j} m}=\frac{k_{m u} / k_{m}}{\rho c} \frac{p_{m}\left(u_{j}\right)}{v_{m}\left(u_{j}\right)}$ at any wall $u_{j}$ for each transversal mode (see details in Appendix):

$$
\zeta_{u_{j} m}=\frac{\xi_{m_{u}}^{(a, l)}+i \xi_{m_{u}}^{(w, l)} \tan \left(k_{m_{u}}^{(w)} h\right)}{1+\xi_{m_{u}}^{(a, w)} \tan \left(k_{m_{u}}^{(w)} h\right)}
$$


Table 1. Physical parameters of the media (speed of sound, density, and effective characteristic impedance).

\begin{tabular}{cccc} 
& $c[\mathrm{~m} / \mathrm{s}]$ & $\rho\left[\mathrm{kg} / \mathrm{m}^{3}\right]$ & $Z=\rho c\left[\mathrm{Ns} / \mathrm{m}^{3}\right]$ \\
\hline Wall (glass) & 5600 & 2500 & $14 \times 10^{6}$ \\
Liquid (water) & 1500 & 1000 & $1.5 \times 10^{6}$ \\
Air & 340 & 1.225 & 416
\end{tabular}

where

$$
\xi_{m_{u}}^{(\alpha, \beta)}=\frac{\rho_{\alpha} c_{\alpha}}{\rho_{\beta} c_{\beta}} \frac{k_{m_{u}}^{(\beta)}}{k_{m}^{(\beta)}} \frac{k_{m}^{(\alpha)}}{k_{m_{u}}^{(\alpha)}}
$$

the superscript $l$ being used here to refer to the liquid $\left(k_{m}^{(l)} \equiv k_{m}, k_{m_{u}}^{(l)} \equiv k_{m_{u}}\right)$.

This impedance $\zeta_{u_{j} m}$ shows a resistive (due to the energy leakage through the wall) and an inertial and elastic behaviour of the boundary, represented by the impedances $\rho_{\alpha} c_{\alpha}$ as expected ( $c_{\alpha}$ depending on the compressibility for the fluid and on the Young modulus for the wall). It is worth noting that in the limit of small thickness $h$ of the wall $\left(\left|k_{m}^{(w)}\right| h \ll 1\right)$, in the lower frequency range and considering the fundamental mode in the wall, $\tan \left(k_{m_{u}}^{(w)} h\right)$ reduces to the real factor $\left(k_{m_{u}}^{(w)} h\right)$. This last approximate expression implies both that the reflected modal pressure wave inside the tank is not exactly, but nearly, out of phase with the incident wave and that the modulus of the reflection coefficient

$$
R_{u_{j} m}=-\frac{1-\zeta_{u_{j} m}}{1+\zeta_{u_{j} m}}
$$

is slightly lower than 1 (energy transfer from liquid inside the tank to air outside through the wall). Furthermore, at the limit $(h=0)$, the reflection coefficient reduces to

$$
R_{u_{j} m}=-\frac{1-\xi_{m_{u}}^{(a, l)}}{1+\xi_{m_{u}}^{(a, l)}},
$$

which is a negative constant whose the modulus is slightly lower than one $\left(\rho_{a} c_{a}\right) /(\rho c) \simeq 3 \times 10^{-4}$ (see table 1). This expression applies at the upper interface between liquid and air. Invoking this expression, one should note also that the zero-order approximation leads to $R_{u_{j} m} \simeq-1$ (Dirichlet condition), which corresponds to a perfectly soft (pressure release) wall. Note that, depending on the frequency and the thickness of the walls $\left(k_{m_{u}}^{(w)} h\right)$, expression (8) represents an impedance-like coefficient which corresponds to boundary conditions extending from nearly Dirichlet conditions to nearly Neumann conditions.

\section{Tank with lossy and reacting walls: eigenfunctions and eigenvalues, normal mode solutions}

In order to express the eigenfunctions and the eigenvalues of the acoustic field in the "brick" of water , the unknown coefficients $B_{m_{x}}, B_{m_{y}}$, and $B_{m_{z}}$ which appear in the modal wave functions (4) can be expressed as functions of the reflection coefficients $R_{u_{j} m}$ (10) as follows [18]:

at the wall $u=0$ labelled "1" (below, $u$ stands for $x$, or $y$, or $z$ indifferently), the reflected amplitude to incident amplitude ratio is,

$$
\frac{1}{B_{m_{u}}}=R_{u_{1} m}
$$

and at the wall $u=L_{u}$ labelled "2", the incident amplitude to reflected amplitude ratio is,

$$
B_{m_{u}} e^{2 i k_{m_{u}} L_{u}}=R_{u_{2} m} .
$$


Then, the individual eigenvalues $k_{m_{u}}$ are roots of the following equation, which is obtained by eliminating $B_{m_{u}}$ between these equations (12) and (13):

$$
e^{2 i k_{m_{u}} L_{u}}=R_{u_{1} m} R_{u_{2} m}
$$

i.e. the roots of

$$
k_{m_{u}} L_{u}=m_{u} \pi-\frac{i}{2} \ln \left(R_{u_{1} m} R_{u_{2} m}\right),
$$

where $m_{u}=0,1,2,3, \cdots, m_{u}=0$ accounting for the uniform pressure field (no velocity field associated with, in the corresponding direction) occurring in the lowest frequency range (below the first resonance frequency), which vanishes when $R_{u_{1} m}=R_{u_{2} m}=-1$.

Invoking the approximate form of equation (15), to the first order of the small factor $\zeta_{u_{j} m}$ (8), it follows that

$$
k_{m_{u}} L_{u} \cong m_{u} \pi+i\left(\zeta_{u_{1} m}+\zeta_{u_{2} m}\right) \text {. }
$$

Finally, writing the coefficient $B_{m_{u}}=1 / R_{u_{1} m}$ (Eq. (12)) in Eq. (4) under the following convenient form and invoking (10),

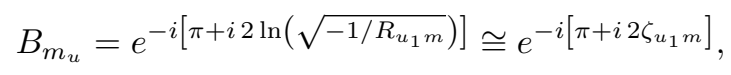

where the last expression is an approximation to the first order in the quantity $R_{u_{1} m}$, which involves the small parameters $\zeta_{u_{1} m}$, the complex expression for the modal solution can be written as

$$
\begin{aligned}
\psi_{m}(x, y, z)= & A_{m} \sin \left(k_{m_{x}} x-i \zeta_{x_{1} m}\right) \\
& \cdot \sin \left(k_{m_{y}} y-i \zeta_{y_{1} m}\right) \\
& \cdot \sin \left(k_{m_{z}} z-i \zeta_{z_{1} m}\right),
\end{aligned}
$$

where $A_{m}=\sqrt{2^{3} /\left(L_{x} L_{y} L_{z}\right)}$ is the normalization factor.

These eigenfunctions approximately satisfy the usual orthogonality property because, for the coordinate $x$ (and similarly for the coordinates $y$ and $z$ ),

$$
\begin{aligned}
0 & \cong \int_{0}^{L_{x}} \partial_{x}\left(\psi_{n_{x}} \partial_{x} \psi_{m_{x}}-\psi_{m_{x}} \partial_{x} \psi_{n_{x}}\right) d x \\
& =\left(k_{m_{x}}^{2}-k_{n_{x}}^{2}\right) \int_{0}^{L_{x}} \psi_{m_{x}} \psi_{n_{x}} d x \\
& =\left(k_{m_{x}}^{2}-k_{n_{x}}^{2}\right) \delta_{m_{x} n_{x}}
\end{aligned}
$$

when using the lower order of the small factor $\zeta_{u_{j} m}$.

Invoking expression (5) with $\omega_{m}=c k_{m}(21)$, the associated solution for the acoustic pressure mode is

$$
\begin{aligned}
p_{m}(x, y, z ; t) & =\psi_{m}(x, y, z) e^{i \omega_{m} t} \\
& =\psi_{m}(x, y, z) e^{i \omega_{m}^{(0)}\left(1-\Xi_{m}^{(i)}\right) t} e^{-\omega_{m}^{(0)} \Xi_{m}^{(r)} t},
\end{aligned}
$$

showing the transient behaviour of each mode.

Using Eq. (18) with the Euler equation, it appears that the particle velocity field is quasi normal to the wall at any location on the wall (the components tangent to the wall are very small).

Note that, invoking the approximate expression of the eigenvalues (16) and equation (2), it follows that

$$
k_{m} \cong k_{m}^{(0)}\left(1+\Xi_{m}\right)=k_{m}^{(0)}\left(1-\Xi_{m}^{(i)}+i \Xi_{m}^{(r)}\right),
$$


with

$$
\begin{aligned}
\Xi_{m}= & \frac{1}{\left(k_{m}^{(0)}\right)^{2}}\left[\frac{m_{x} \pi}{L_{x}^{2}}\left(\zeta_{x_{1} m}+\zeta_{x_{2} m}\right)\right. \\
& \left.+\frac{m_{y} \pi}{L_{y}^{2}}\left(\zeta_{y_{1} m}+\zeta_{y_{2} m}\right)+\frac{m_{z} \pi}{L_{z}^{2}}\left(\zeta_{z_{1} m}+\zeta_{z_{2} m}\right)\right],
\end{aligned}
$$

the corresponding angular frequency being

$$
\begin{aligned}
\omega_{m} & =c k_{m} \simeq \omega_{m}^{(0)}\left(1+i \Xi_{m}\right) \\
& =\omega_{m}^{(0)}\left(1-\Re\left(\Xi_{m}\right)+i \Im\left(\Xi_{m}\right)\right) .
\end{aligned}
$$

Losses inside both the water and the walls, which are small compared to those due to the leakage through the walls that are involved above in the modelling, are not considered in this above modelling so that results may not be wholly accurate, especially at the resonances because their level is very sensitive to any small dissipation factor. An efficient but simple procedure for taking into account these minor (but non-negligible) losses thus warrants consideration. The simple and usual procedure is to introduce this minor additional absorption by substituting the complex wavenumbers $k^{*}$ and $k_{w}^{*}$ (respectively in the water and the wall) for $k$ and $k_{w}$, or equivalently by using a complex speed of sound $c^{*}$ and $c_{w}^{*}$ (associated respectively to complex compressibility and Young modulus) such that $k^{*} / k=c / c^{*}=1+i \delta$ and $k_{w}^{*} / k_{w}=c_{w} / c_{w}^{*}=1+i \delta_{w}$, the imaginary parts $\delta$ and $\delta_{w}$ representing the small attenuation of the waves. It appears in fact that, in the frequency range of interest $(f<10 \mathrm{kHz})$, the effects of the losses in the water are negligible [19] compared to those in the glass [20, 21]. So, the only minor additional absorption that should be accounted for is the one in the wall represented by the parameter $\delta_{w}$. Because both, the precise nature of the walls and the damping at their edges are unknown, the value of $\delta_{w}$ should be fitted: we found $\delta_{w}=0.022$ from the transient behaviour of the pressure field when a sinusoidal excitation is shut off (Fig. 4), arbitrarily in the tank n.2 (experiment 2 in table 2).

This semi-empirical method appears to be reliable since it provides accurate results (see section 5) with an approximately known value $\delta_{w}$ which is independent of the frequency (the same value for all the resonances considered), and moreover, it is of the same order of magnitude as those found in literature [21]. Note that, besides, due to the unknown precise nature of the glass, its other parameters (density and speed of sound) given in table 1 are not very accurate because they are rounded to two significant figures.

\section{Tank with lossy and reacting walls: normal mode solutions to the acoustic problem in the liquid-filled rectangular tank when a compressional localized source provides energy.}

Within the tank, the pressure variation is assumed to satisfy an inhomogeneous propagation equation, with a harmonic (angular frequency $\omega$ ) quasi point-source term on the right-hand side. The construction of the solution in terms of eigenmodes of the tank (modal expansion of the Green function in the frequency domain) makes use of the modal wave functions (18) with the eigenvalues (21). Then, the inverse Fourier Transform provides the Green function of the problem considered in the time domain (which is usually the primarily desired descriptor).

The Helmholtz equation is then written as follows:

$$
\left[\partial_{x x}^{2}+\partial_{y y}^{2}+\partial_{z z}^{2}+k^{2}\right] P(x, y, z ; \omega)=-\delta\left(x_{0}, y_{0}, z_{0}\right),
$$

where $\delta\left(x_{0}, y_{0}, z_{0}\right)$ is the Dirac function and $k=\omega / c$. The pressure field is then expressed as the well-known Green function in the Fourier domain

$$
P(x, y, z ; \omega)=\sum_{m=0}^{\infty} \frac{\psi_{m}\left(x_{0}, y_{0}, z_{0}\right)}{k_{m}^{2}-k^{2}} \psi_{m}(x, y, z),
$$


Table 2. Dimensions of the water-filled tanks and position of the hydrophones for each experiment. The first and second dimensions denote the length and the width of the water in the tank, the third one denotes the depth (water level).

\begin{tabular}{cccc} 
& Exp. 1 & Exp. 2 & Exp. 3 \\
\hline Tank dimensions $[\mathrm{cm}]$ & $49 \times 23.9 \times 13.9$ & $58.6 \times 28.9 \times 18.4$ & $39.4 \times 19.3 \times 13.7$ \\
Wall thickness $h[\mathrm{~mm}]$ & 2.7 & 3.8 & 2.7 \\
Source position $[\mathrm{cm}]$ & {$[33,13,11]$} & {$[27,8,8]$} & {$[21.5,10,8.5]$} \\
Receiver position $[\mathrm{cm}]$ & {$[20,15,11]$} & {$[23.5,1514]$} & {$[30,13.5,9]$}
\end{tabular}

( $m=0$ meaning $m_{x}, m_{y}, m_{z}=0$, the eigenfunctions $\psi_{m}$ being assumed to be normalized) leading to the harmonic solution $p(x, y, z ; t)=P(x, y, z ; \omega) e^{i \omega t}$. The associated standing velocity wave is obtained readily from Euler's equation $\rho \partial_{t} \vec{v}=-\vec{\nabla} p$.

The coefficients $C_{m}$ remain finite everywhere on the $\omega$-axis because the eigenvalues $k_{m}$ are complex and the wavenumber $k=\omega / c$ is real, and, owing to the fact that the damping and reactive factors at the walls of the tank are very small, these coefficients are very small everywhere on the $\omega$-axis except when the angular frequency considered is very close to the real part of an eigenfrequency $\omega_{m}=c \operatorname{Re}\left(k_{m}\right)$. One should note that with such a construction of the solution (modal expansion), the satisfaction of the boundary conditions follows automatically from the fact that each of the eigenfunctions satisfies these boundary conditions, and that the set of eigenfunctions is a complete set. Moreover, because the coefficients of the expansion behave asymptotically as $\left(1 / m_{x}^{2}\right),\left(1 / m_{y}^{2}\right)$, and $\left(1 / m_{z}^{2}\right)$, and because the eigenfunctions are bounded, the series $(25)$ is absolutely and uniformly convergent. It converges towards the continuous function which represents the acoustic pressure field in the intervals considered.

Any solution in the time domain takes the form of a convolution product of a source function $f(x, y, z ; t)$ in the time domain and the impulse response of the tank (the inverse Fourier transform of the Green function (25), which can be handled numerically). This quite simple result (convolution product) occurs because the pressure variation and the Green's function satisfy here the same boundary conditions. It is worth noting that the considered source function must behave as a distribution of quasi point-sources and that an extended source will have to be taken into account in the formalism as a moving boundary of the domain.

Note that when the source is a vibrating source, the inverse Fourier transform of the source term on the righthand side of the propagation equation takes the following form

$$
f(x, y, z ; t)=\rho \partial_{t} q(x, y, z ; t)
$$

where $q(x, y, z ; t)\left[\mathrm{s}^{-1}\right]$ is the rate of flow of water away from an elementary source, in every direction, divided by the volume of the source (volume velocity per unit volume). As an example, for a simple spherical source of small radius $a \rightarrow 0$ (monopole), the volume velocity $q=4 \pi a^{2} v /\left(\frac{4}{3} \pi a^{3}\right)$ is the instantaneous value of the total flow of water away from the centre of the localized spherical source (flow outward from the origin of the source) divided by the volume of the source, when the radius is small compared with the wavelength of the sound radiated.

\section{Experimental verification and results}

\subsection{Experimental verification}

In the experimental set-up, two Brüel \& Kjær Hydrophones Type 8103 are used for measurements in several rectangular water-filled tanks, the first one being used as a source to generate the acoustical waves (amplified by a 


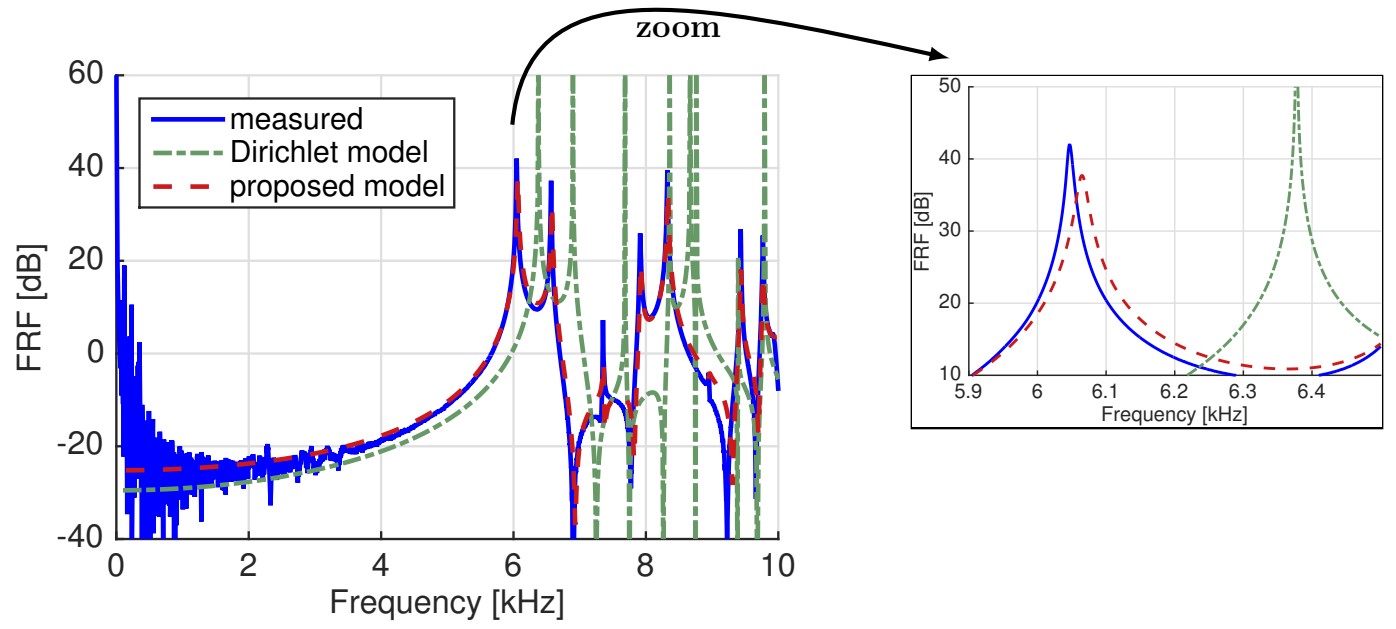

Figure 1. (colour online) Measured (blue solid line), Dirichlet model (green dash-dotted line), and proposed model (red dashed line) FRF between source (coordinates [33, 13, 11] cm) and receiver (coordinates $[20,15,11] \mathrm{cm}$ ) in the tank with dimensions $49 \mathrm{~cm} \times 23.9 \mathrm{~cm} \times 13.9 \mathrm{~cm}$.

Brüel \& Kjær Power Amplifier Type 2713), the second one being used as a receiver. The signals are generated and acquired using Matlab and an RME Fireface 400 interface.

As a first application of the model discussed above, its predictions in terms of the modal behaviour of the acoustic pressure fields are compared with experimental results in three water-filled tanks with different dimensions, wall thicknesses, and positions of the hydrophones (see table 2). The material constants are provided in table 1. A swept-sine signal $s(t)$ (Fourier Transform of $s(t)$ being denoted below $S(\omega)$ ) [22], ranging from $10 \mathrm{~Hz}$ to $10 \mathrm{kHz}$, is sent through the Brüel \& Kjær Power Amplifier Type 2713 to the emitting hydrophone Brüel \& Kjær Type 8103 placed at the source position according to table 2. Another (receiving) hydrophone of the same type is placed at the receiver position according to table 2 and used to acquire the measurement signal $r(t)$ (Fourier Transform of $r(t)$ being denoted below $R(\omega)$ ). While the sensitivity of the receiving hydrophone B\&K Type 8103 is flat vs. frequency from $0.1 \mathrm{~Hz}$ to $20 \mathrm{kHz}(+1 /-1.5 \mathrm{~dB})$, the efficiency of the emitting hydrophone as a function of the frequency has a $+12 \mathrm{~dB} /$ octave slope (typical for voltage-driven piezoelectric hydrophones) that must be compensated for numerically in the data post-processing (by $\omega^{-2}$ factor). It is worth noting that the unknown source efficiency $A_{0}$ (including the amplifier) has also been evaluated to adjust the theoretical and experimental results in the lower frequency range. Finally, Frequency Response Function (FRF) $H(\omega)$ between the source and the receiver is calculated as

$$
H(\omega)=\frac{A_{0}}{\omega^{2}} \frac{R(\omega)}{S(\omega)} .
$$

In Figs. 1, 2, and 3 (color online), the experimental results calculated using Eq. (27) (blue solid line) are compared with both the model in which Dirichlet boundary conditions are used (Eq. (2), green dash-dotted line) and the model proposed in this paper using Eq. (25) (red dashed line). The model appears to be well suited for interpreting the experimental results in any rectangular fluid-filled tank: the FRF estimated using the proposed model agree much better with the measured FRF than the "Dirichlet model" in all the experiments.

Note that the maximal value of each integer $m_{x}, m_{y}, m_{z}$ is here equal to 16 and that the computational time is always lower than $0.4 \mathrm{~s}$ (computations have been run on a personal laptop with $16 \mathrm{~Gb}$ of random access memory and with an Intel Core i7 processor with 4 cores running at 2,2 $\mathrm{GHz}$ and with $16 \mathrm{MB}$ of cache memory).

The second application of the model presented here concerns the transient behaviour of the pressure field when a sinusoidal excitation is shut off. The configuration denoted Exp. 2 in table 2 is used. The frequency of the 


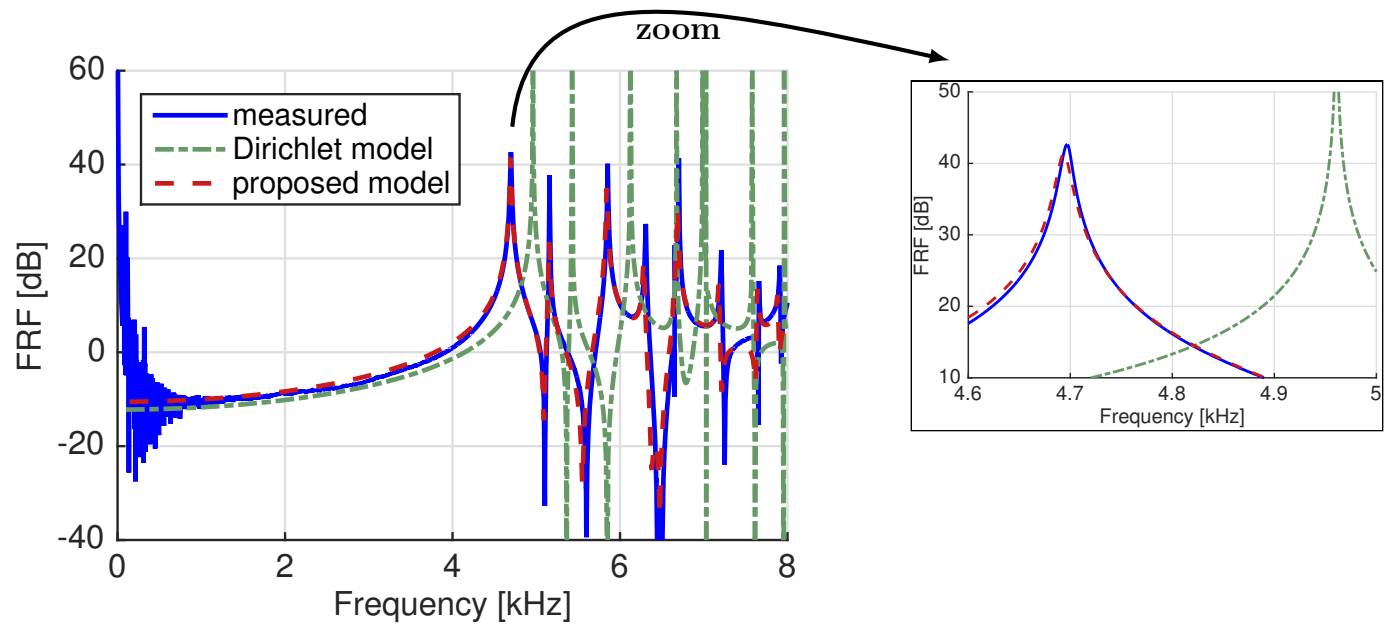

Figure 2. (colour online) Measured (blue solid line), Dirichlet model (green dash-dotted line), and proposed model (red dashed line) FRF between source (coordinates $[27,8,8] \mathrm{cm}$ ) and receiver (coordinates $[23.5,1514] \mathrm{cm}$ ) in the tank with dimensions $58.6 \mathrm{~cm} \times 28.9 \mathrm{~cm} \times 18.4$.

sinusoidal power source is chosen very close to the resonance frequency of the mode $[1,1,1]$ and therefore, when this power source, driving a particular standing wave, is shut off, the wave will damp out exponentially (Eq. (20)). In order to emphasize this property, the transient response (sound-decay curve, acoustic level versus time) is depicted with a logarithmic scale in Fig. 4 (color online). Experimental (blue solid line) and theoretical (red dashed line) results coincide very closely and lead to the reverberation time of $\mathrm{RT}_{60}=0.22 \mathrm{~s}$ (the length of time for the level to drop $60 \mathrm{~dB}$ ). Note that the Dirichlet model leads to an infinite reverberation time (the waves do not damp out).

One should note that the modes can be separated into two categories: those whose at least one index is zero and those whose the three indexes are all different from zero. Only the later contribute to the resonances that appear on Figs. 1-3. For example, the five modes which correspond to the five resonances in Fig. 3 are: $(1,1,1)$ at $6551 \mathrm{~Hz}$, $(2,1,1)$ at $7276 \mathrm{~Hz},(3,1,1)$ at $8352 \mathrm{~Hz},(1,2,1)$ at $9070 \mathrm{~Hz}$, and $(2,2,1)$ at $9620 \mathrm{~Hz}$. The other twenty-four modes in the frequency range $(0-10 \mathrm{kHz})$ have at least one zero index and, as a result, their amplitudes are very low (see Eqs. $(18,21))$. Eleven of them have an eigenfrequency lower than the first resonant frequency $(6551 \mathrm{~Hz})$.

\subsection{Sound field level as a function of distance between emitting and receiving transducers}

The following theoretical results are obtained using the water-filled tank model proposed in this paper (Eqs. (18) and (25)) to illustrate the propagation of acoustic waves as a function of frequency and source/receiver positions (the distance between them). The source is placed at coordinates $[10,15,10] \mathrm{cm}$ in a tank with dimensions $60 \mathrm{~cm}$ x $30 \mathrm{~cm} \times 20 \mathrm{~cm}$ (the third dimension denoting the water level) and the wall thickness $h=2.7 \mathrm{~mm}$. The FRF (Fig. 5(a)) between the source and the receiver is then estimated for different coordinates of the receiver $\left[x_{r}, 15\right.$, 10] $\mathrm{cm}$, where $x_{r}$ varies from $10 \mathrm{~cm}$ to $50 \mathrm{~cm}$ (see Fig. 5(b)). This estimated FRF shows a behaviour at frequencies below the resonance frequency (e.g. $1 \mathrm{kHz}$ ) that is very different from that at the resonance frequency $(4.7 \mathrm{kHz})$. In Fig. 5(a), the level of the FRF is depicted for these two frequencies using black solid lines (with circle-marks at $1 \mathrm{kHz}$ and with x-marks at $4.7 \mathrm{kHz}$ ) and these two lines are plotted again in Fig. 5(c). While the level of the first resonance is almost independent of the position of the receiver, the level of the FRF at $1 \mathrm{kHz}$ decreases drastically while the receiver moves away from the source. Accordingly, the acoustic field provided by a source, with energy distributed mainly below the resonance frequency, will be very quickly attenuated with the distance from the source. To verify these theoretical results experimentally, measurements using a sine wave were performed for frequencies 


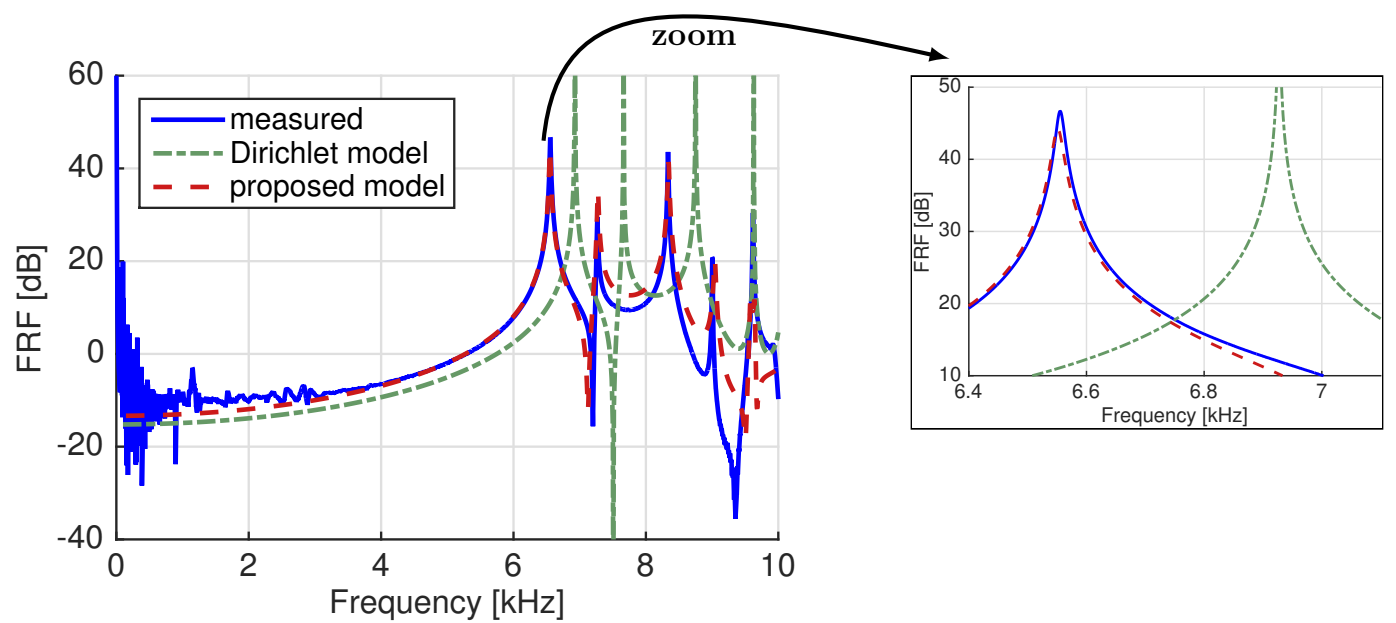

Figure 3. (colour online) Measured (blue solid line), Dirichlet model (green dash-dotted line), and proposed model (red dashed line) FRF between source (coordinates $[21.5,10,8.5] \mathrm{cm}$ ) and receiver (coordinates [30, 13.5, 9] $\mathrm{cm}$ ) in the tank with dimensions $39.4 \mathrm{~cm} \times 19.3 \mathrm{~cm} \times 13.7 \mathrm{~cm}$.

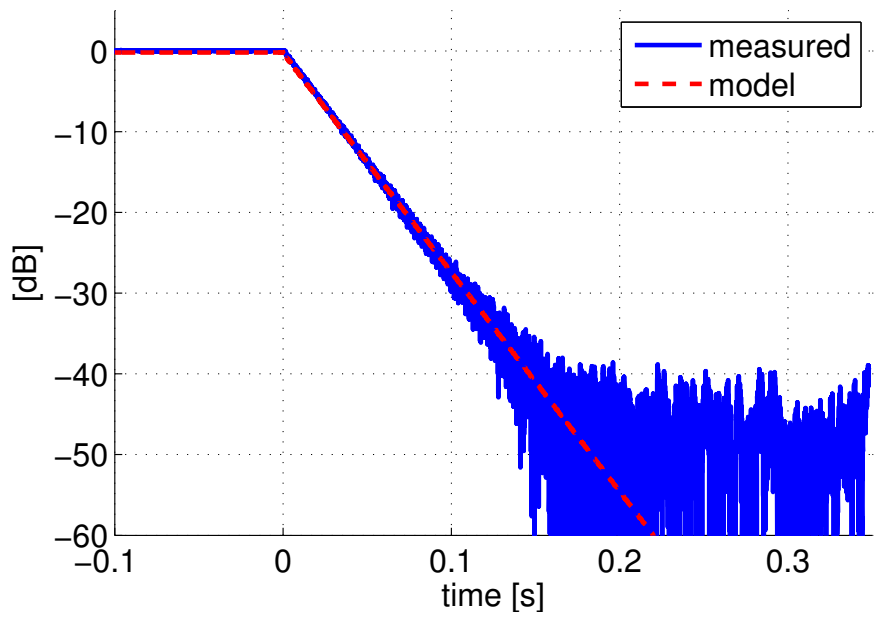

Figure 4. (colour online) Measured (blue solid line) and proposed model (red dashed line) envelope of the transient response, after the sinusoidal source signal (frequency $4.7 \mathrm{kHz}$ ) is shut off. Source and receiver are placed at coordinates $[27,8,8] \mathrm{cm}$ and $[23.5,1514] \mathrm{cm}$ respectively in the tank with dimensions $58.6 \mathrm{~cm} \mathrm{x} 28.9 \mathrm{~cm} \times 18.4$ $\mathrm{cm}$.

of $1 \mathrm{kHz}$ and $4.7 \mathrm{kHz}$. The results plotted in Fig. 5(c) (*-marks for $1 \mathrm{kHz}$ and dot marks for $4.7 \mathrm{kHz})$ show that the experimental decreasing vs. distance at low frequencies (at $1 \mathrm{kHz}$ ) is very close to the theoretical one. The level at the resonant frequency $(4.7 \mathrm{kHz})$ obtained experimentally shows slight discrepancies that have the same order as those on Figs. 1, 2, and 3. Note also, that similar behaviour has been experimentally observed in [4]. The reason for such behaviour comes from the nature of the boundary conditions at the tank walls, which are close to the Dirichlet boundary conditions. This fact leads to emitted pressure waves that are, in the lower frequency range, reflected with almost the same amplitude, but with nearly inverted phase. Then, the superposition of the direct and reflected waves almost vanishes. Note also, that the theoretical curve (Fig. 5(c)) is obtained using limited number of modes $\left[\operatorname{Max}\left(m_{x}, m_{y}, m_{z}\right)=16\right]$. Increasing the number of modes can lead to a slightly better match at resonance frequencies, but at the cost of computational time. 


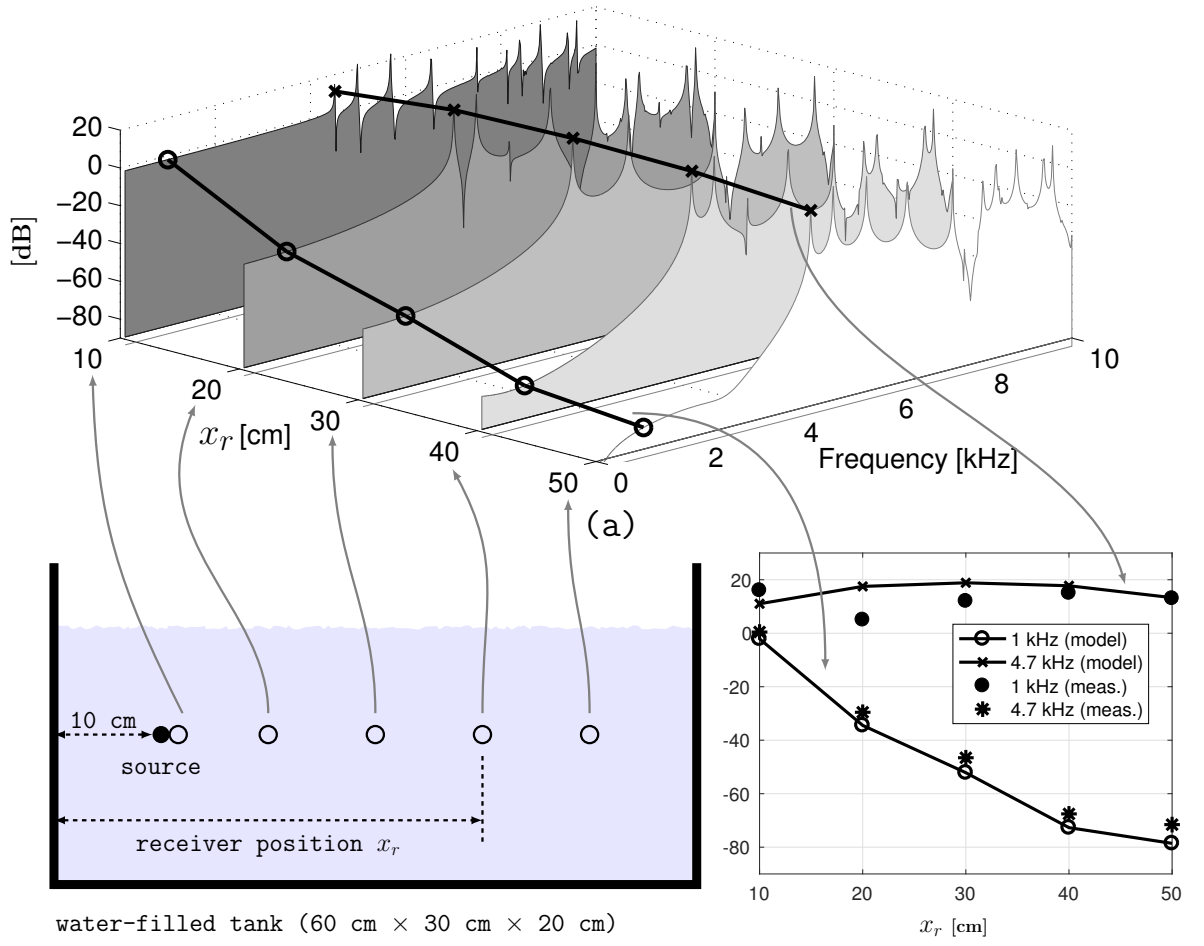

(b)

(c)

Figure 5. Sound field level as a function of the distance between emitting and receiving transducers. (a) theoretical FRFs, (b) schematic representation of the hydrophone positioning in the tank, (c) theoretical and experimental results compared for $1 \mathrm{kHz}$ and $4.7 \mathrm{kHz}$.

\subsection{Pressure and Velocity field distribution}

The theoretical results proposed in this subsection rely on the three-dimensional mapping of both the modulus of the pressure field and the vector velocity field for the mode [2,2,1] given in Fig. 6 (color online). This mapping shows several expected features. First, owing to the fact that the boundary condition at the top water-air interface $(\mathrm{z}=0.2 \mathrm{~m})$ is very close to the Dirichlet (pressure-release) condition for which the pressure is zero at the boundary, the pressure field is almost equal to zero $(-80 \mathrm{~dB})$ at that interface. Second, the pressure field values on the water-wall boundaries [e.g. $\mathrm{z}=0$ Fig. $6(\mathrm{a})$, or $\mathrm{y}=0$ Fig. 6(b)] are much higher than at the top water-air interface, reaching non-negligible values $(-45 \mathrm{~dB}$ to $-20 \mathrm{~dB}$ from the maximal pressure inside the tank), showing that the pressure-release $(\mathrm{p}=0)$ boundary condition $[1,2,3,4]$ is only a first-order approximation. Third, as mentioned above [discussions that follows Eq. (3) and (20)], Fig. 6 shows that, on the boundaries, the velocity field depicted with grey cones is almost perpendicular to the boundary interface (its tangential component vanishes) even though the viscous effects are neglected.

\section{Conclusion}

An approximate analytical model for describing the acoustic field inside a small-sized fish tank has been worked out, which accounts for the damping and phase shift due to the reflections on the six surfaces of the "brick" of water. The model is suited for interpreting, in a very simple way, the acoustic field, both pressure field and particle velocity (or displacement) field, when a compressional power source provides energy and when the source is shut off. Its predictions should be useful for improving the description of acoustic phenomena encountered in current applications. As a preliminary test of the effectiveness of the model, it was used to predict salient acoustic properties 

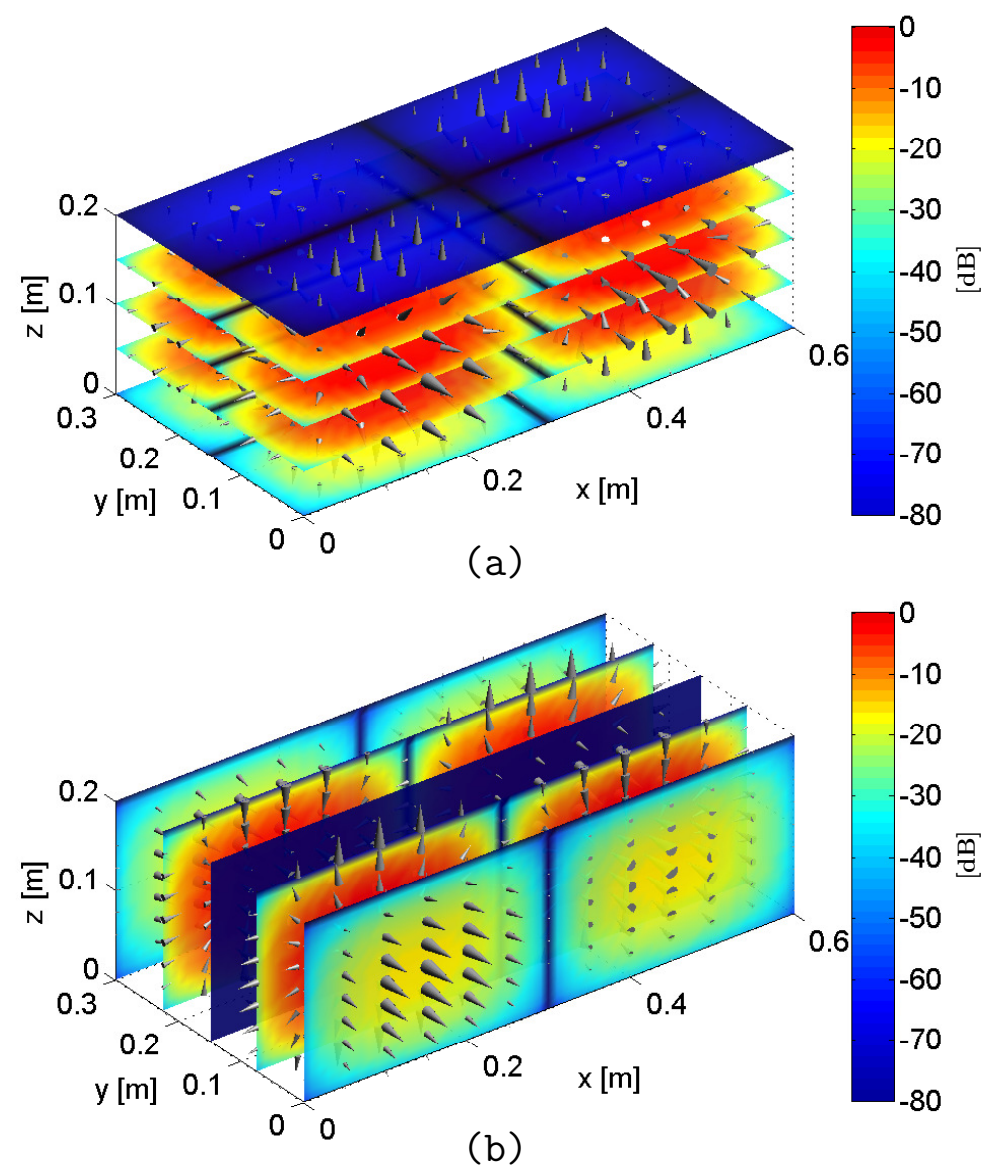

Figure 6. (colour online) Three dimensional mapping of both the modulus of the pressure field (colours) and the vector velocity field, for the mode $[2,2,1]$ (theoretical results).

of devices, emphasized with the help of the experimental results obtained here (two of them being considered previously in the literature [4]). In both cases (stationary and transient fields), an overall satisfactory agreement was found between the results calculated and the experimental ones, providing confidence in the correctness of the derived solutions and their implementation. This good agreement results from the fact that the constant parameter delta, which represent the small dissipation effect that is added to the main dissipation effect (energy transfer to the air), was chosen to fit very accurately the quality factors of the resonances. In fact, the decay curve can be used to adjust this small parameter and to verify that its value matches the values known in the literature (as we verified above).

As mentioned in the literature [4, introduction], several materials for sound absorption placed on the walls (fibres, sand, ...) have been used to reduce reverberation and therefore to reduce the amplitude of the modes in the tank. So far, to our knowledge, no theoretical attempt has been carried out to interpret the efficiency, in terms of absorption of energy in a liquid-filled tank. On the other hand, in the domain of air-filled cavities, authors show the effects of irregular shapes (tilted wall) on the eigenmodes (and their coupling) and their usefulness for such perturbed fields $[13,23]$. These two techniques are likely to be the subject of investigations (among others) in the near future. These models, derived from the models proposed in the present paper, might improve our understanding of the acoustic fields in such coated fluid-filled tanks with or without tilted boundary surfaces, which are more appropriate than the fields in rectangular non-coated tanks. 


\section{Acknowledgements}

This research was funded by the Region Pays de la Loire within the Le Mans Acoustic Project. The authors are indebted to Marcel Koken from LABOCEA, Brest, France and to Petr Cisar from the ICS, University of South Bohemia in Ceske Budejovice, Czech Republic, for substantial help in providing respectively the early subject matter of the project and experimental facilities. They express their gratitude to Dr. Eric Bavu (CNAM, Paris, France), Prof. Laurent Simon (LAUM, Le Mans, France) for helpful discussions.

\section{References}

[1] A Parvulescu. Problems of propagation and processing. In Tavolga WN (ed) Marine bio-acoustics, pages 87-100. Pergamon, Oxford, 1964.

[2] A Parvulescu. The acoustics of small tanks. In Tavolga WN (ed) Marine bioacoustics II, pages 7-13. Pergamon, Oxford, 1967.

[3] Tsuyoshi Okumura, Tomonari Akamatsu, and Hong Y Yan. Analyses of small tank acoustics: empirical and theoretical approaches. Bioacoustics, 12(2-3):330-332, 2002.

[4] Tomonari Akamatsu, Tsuyoshi Okumura, Nicola Novarini, and Hong Y Yan. Empirical refinements applicable to the recording of fish sounds in small tanks. The Journal of the Acoustical Society of America, 112(6):3073$3082,2002$.

[5] Chan-Yi Liao, Yi-Chuang Wu, Ching-Yuan Chang, and Chien-Ching Ma. Theoretical analysis based on fundamental functions of thin plate and experimental measurement for vibration characteristics of a plate coupled with liquid. Journal of Sound and Vibration, 394:545 - 574, 2017.

[6] Alec J. Duncan, Klaus Lucke, Christine Erbe, and Robert D. McCauley. Issues associated with sound exposure experiments in tanks. In Proceedings of Meetings on Acoustics 4enal, volume 27. ASA, 2016.

[7] Rui Tang, Yiming Zhang, Qi Li, and Dajing Shang. The investigation of the methods for predicting the sound field in a non-anechoic tank with elastic boundary. In Ocean Acoustics (COA), 2016 IEEE/OES China, pages 1-6. IEEE, 2016.

[8] Philip Morse and K Uno Ingard. Theoretical acoustics. Princeton university press, 1968.

[9] Michel Bruneau and Thomas Scelo (translator and contributor). Fundamentals of acoustics. ISTE, London, 2006.

[10] Antoine Chaigne and Jean Kergomard. Acoustique des instruments de musique (2e édition revue et augmentée). Belin, 2013.

[11] Sylvio R. Bistafa and John W. Morrissey. Numerical solutions of the acoustic eigenvalue equation in the rectangular room with arbitrary (uniform) wall impedances. Journal of Sound and Vibration, 263(1):205-218, 2003.

[12] Yusuke Naka, Assad A. Oberai, and Barbara G. Shinn-Cunningham. Acoustic eigenvalues of rectangular rooms with arbitrary wall impedances using the interval newton/ generalized bisection method. The Journal of the Acoustical Society of America, 118(6):3662-3671, 2005.

[13] Y. Li and L. Cheng. Vibro-acoustic analysis of a rectangular-like cavity with a tilted wall. Applied Acoustics, 68(7):739-751, 2007. 
[14] Nobuo Tanaka, Yusuke Takara, and Hiroyuki Iwamoto. Eigenpairs of a coupled rectangular cavity and its fundamental properties. The Journal of the Acoustical Society of America, 131(3):1910-1921, 2012.

[15] Jie Pan and David Alan Bies. The effect of fluid-structural coupling on sound waves in an enclosure theoretical part. The Journal of the Acoustical Society of America, 87(2):691-707, 1990.

[16] Guoyong Jin, Shuangxia Shi, and Zhigang Liu. Acoustic modeling of a three-dimensional rectangular opened enclosure coupled with a semi-infinite exterior field at the baffled opening. The Journal of the Acoustical Society of America, 140(5):3675-3690, 2016.

[17] Mirosław Meissner. Acoustics of small rectangular rooms: Analytical and numerical determination of reverberation parameters. Applied Acoustics, 120:111-119, 2017.

[18] Michel Bruneau, Philippe Gatignol, Patrick Lanceleur, and Catherine Potel. Exercices d'Acoustique Tome 1. Cépaduès-Editions Toulouse, 2016. Chapter 2.

[19] Michael A. Ainslie and James G. McColm. A simplified formula for viscous and chemical absorption in sea water. The Journal of the Acoustical Society of America, 103(3):1671-1672, 1998.

[20] Michael Vorländer. Auralization: fundamentals of acoustics, modelling, simulation, algorithms and acoustic virtual reality. Springer Science \& Business Media, 2007.

[21] J.J. Mills. Low frequency storage and loss moduli of soda-silica glasses in the transformation range. Journal of Non-Crystalline Solids, 14(1):255 - 268, 1974.

[22] Antonin Novak, Pierrick Lotton, and Laurent Simon. Synchronized swept-sine: Theory, application, and implementation. Journal of the Audio Engineering Society, 63(10):786-798, 2015.

[23] Pascal Hamery, Philippa Dupire, and Michel Bruneau. Acoustic fields in trapezoidal cavities. Acta Acustica united with Acustica, 83(1):13-18, 1997. 


\section{Appendix}

\section{A.1 The behaviour of the walls : the modal impedance-like, the quasi flexural wave of a supported plate}

The pressure wave function and the $u$ - component of the particle velocity fields (obtained from the Euler equation $\left.\rho \partial_{t} \vec{v}=-\vec{\nabla} p\right)$, inside the tank, in the wall, and in the air outside the tank, can be written as follows, with $\omega_{m}=k_{m} / c=k_{m}^{(w)} / c_{w}=k_{m}^{(a)} / c_{a}$,

\section{- inside the tank}

$$
\left\{\begin{array}{l}
p_{m}(u, t)=A\left(e^{-i k_{m_{u}} u}+R_{u_{j} m} e^{i k_{m_{u}} u}\right) \phi_{\mu_{u}}\left(u^{\prime}, u^{\prime \prime}\right) e^{i \omega_{m} t} \\
v_{m}(u, t)=\frac{A}{\rho c} \frac{k_{m_{u}}}{k_{m}}\left(e^{-i k_{m_{u}} u}-R_{u_{j} m} e^{i k_{m_{u}} u}\right) \phi_{\mu_{u}}\left(u^{\prime}, u^{\prime \prime}\right) e^{i \omega_{m} t},
\end{array}\right.
$$

where $R_{u_{j} m}$ is the reflection coefficient and where the function $\phi_{\mu_{u}}\left(u^{\prime}, u^{\prime \prime}\right)$ represents the modal solution for the acoustic field along the coordinates $u^{\prime}$ and $u^{\prime \prime}$ (transversal modes, $\mu_{u}$ standing for the couple $m_{u}^{\prime}$ and $m_{u}^{\prime \prime}$ ),

- in the wall

$$
\left\{\begin{aligned}
p_{m}^{(w)}(u, t)= & {\left[C_{w} \cos \left(k_{m_{u}}^{(w)} u\right)+D_{w} \sin \left(k_{m_{u}}^{(w)} u\right)\right] } \\
& \phi_{\mu_{u}}^{(w)}\left(u^{\prime}, u^{\prime \prime}\right) e^{i \omega_{m} t} \\
v_{m}^{(w)}(u, t)= & \frac{i}{\rho_{w} c_{w}} \frac{k_{m_{u}}^{(w)}}{k_{m}^{(w)}}\left[-C_{w} \sin \left(k_{m_{u}}^{(w)} u\right)+D_{w} \cos \left(k_{m_{u}}^{(w)} u\right)\right] \\
& \phi_{\mu_{u}}^{(w)}\left(u^{\prime}, u^{\prime \prime}\right) e^{i \omega_{m} t},
\end{aligned}\right.
$$

- in the air outside the tank

$$
\left\{\begin{array}{l}
p_{m}^{(a)}(u=h, t)=A_{a} \phi_{\mu_{u}}^{(a)}\left(u^{\prime}, u^{\prime \prime}\right) e^{i \omega_{m} t} \\
v_{m}^{(a)}(u=h, t)=\frac{A_{a}}{\rho_{a} c_{a}} \frac{k_{m_{u}}^{(a)}}{k_{m}^{(a)}} \phi_{\mu_{u}}^{(a)}\left(u^{\prime}, u^{\prime \prime}\right) e^{i \omega_{m} t},
\end{array}\right.
$$

where $\phi_{\mu_{u}}^{(w)}\left(u^{\prime}, u^{\prime \prime}\right)$ and $\phi_{\mu_{u}}^{(a)}\left(u^{\prime}, u^{\prime \prime}\right)$ are unknown functions, $(\rho$ and $c),\left(\rho_{w}\right.$ and $\left.c_{w}\right)$, and $\left(\rho_{a}\right.$ and $\left.c_{a}\right)$ being the density and the speed of sound in the liquid, the wall, and the air respectively.

The eigenfunctions $\phi_{\mu_{u}}\left(u^{\prime}, u^{\prime \prime}\right)$ and more importantly here the corresponding eigenvalues can be here approximated by those satisfying the Dirichlet conditions (the small particle velocity normal to the walls is assumed to vanish) because, in the expression of the modal impedance-like coefficient Eq. (8) the eigenvalues occur in the upper order terms only. Therefore, one can write there:

$$
\phi_{\mu_{u}}\left(u^{\prime}, u^{\prime \prime}\right)=\sin \left(\frac{m_{u^{\prime}} \pi}{L_{u^{\prime}}} u^{\prime}\right) \sin \left(\frac{m_{u^{\prime \prime}} \pi}{L_{u^{\prime \prime}}} u^{\prime \prime}\right)
$$

with

$$
k_{m}^{2}=k_{m_{u}}^{2}+\left[\left(\frac{m_{u^{\prime}} \pi}{L_{u^{\prime}}}\right)^{2}+\left(\frac{m_{u^{\prime \prime}} \pi}{L_{u^{\prime \prime}}}\right)^{2}\right] .
$$

The continuity of pressure variation and normal particle velocity, respectively at the inner surface $u=0$ and at the outer one $u=h$ of the wall, and at every value of the coordinates $u^{\prime}$ and $u^{\prime \prime}$ on the interfaces, requires that

$$
\begin{gathered}
\phi_{\mu_{u}}\left(u^{\prime}, u^{\prime \prime}\right)=\phi_{\mu_{u}}^{(w)}\left(u^{\prime}, u^{\prime \prime}\right)=\phi_{\mu_{u}}^{(a)}\left(u^{\prime}, u^{\prime \prime}\right), \\
\left(k_{m_{u}}^{(w)}\right)^{2}=\left(k_{m}^{(w)}\right)^{2}-\left[\left(\frac{m_{u^{\prime}} \pi}{L_{u^{\prime}}}\right)^{2}+\left(\frac{m_{u^{\prime \prime}} \pi}{L_{u^{\prime \prime}}}\right)^{2}\right],
\end{gathered}
$$




$$
\left(k_{m_{u}}^{(a)}\right)^{2}=\left(k_{m}^{(a)}\right)^{2}-\left[\left(\frac{m_{u^{\prime}} \pi}{L_{u^{\prime}}}\right)^{2}+\left(\frac{m_{u^{\prime \prime}} \pi}{L_{u^{\prime \prime}}}\right)^{2}\right],
$$

and what follows readily form Eqs. (A.1) to (A.3) is the resulting complex modal impedance-like (8).

The wavenumber $k_{m_{u}}^{(w)}$ can be real (lower order lateral modes, counter-propagative waves in the wall) or imaginary (higher order lateral modes, evanescent waves from each inner surface $u=0$ and $u=h$ in the wall). In the latter case, it is written as

$$
k_{m_{u}}^{(w)}=-i \sqrt{-\left(k_{m}^{(w)}\right)^{2}+\left[\left(\frac{m_{u^{\prime}} \pi}{L_{u^{\prime}}}\right)^{2}+\left(\frac{m_{u^{\prime \prime}} \pi}{L_{u^{\prime \prime}}}\right)^{2}\right]},
$$

and the same for $k_{m_{u}}^{(a)}$.

A relevant point is that expression (A.2) of the particle velocity (and then of the particle displacement) of the wall vanishes when $u^{\prime}$ and $u^{\prime \prime}$ are equal to 0 or to $L_{u^{\prime}}$ or $L_{u^{\prime \prime}}$ respectively (see equations (A.4) and (A.6)). These results mean that the boundaries of the wall do not vibrate (the transverse displacement is equal to zero on the edges of the walls); this is a realistic boundary condition. Moreover, as long as $\left|k_{w} h\right| \ll 1$ both faces of the wall have nearly the same movement (the same transverse displacement). In addition, the transverse behaviour (along $u^{\prime}$ and $u^{\prime \prime}$ ) is coherent with the acoustic field in the liquid near the wall (Eqs. (A.6-A.7)). All these properties show that the solutions (A.2) represent the forced complex waves which clearly reduce nearly (exactly if $h \rightarrow 0$ ) to forced usual flexural waves of a plate simply supported all around. 